\title{
Osteoporotic fracture trends in a population of US managed care enrollees from 2007 to 2017
}

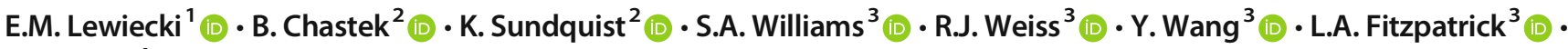 \\ J.R. Curtis ${ }^{4}$ (D)
}

Received: 29 August 2019 / Accepted: 5 February 2020/Published online: 15 February 2020

(C) The Author(s) 2020

\begin{abstract}
Summary This study expands on previous findings that hip fracture rates may no longer be declining. We found that age- and sex-adjusted fracture rates in the US plateaued or increased through mid-2017 in a population of commercially insured and Medicare Advantage health plan enrollees, in contrast to a decline from 2007 to 2013.

Introduction The purpose of this study was to evaluate fracture trends in US commercial and Medicare Advantage health plan members aged $\geq 50$ years between 2007 and 2017 .

Methods Retrospective analysis of the Optum Research Database from January 1, 2007, to May 31, 2017.

Results Of 1,841,263 patients identified with an index fracture, 930,690 were case-qualifying and included in this analysis. The overall age- and sex-adjusted fracture rate decreased from 14.67/1000 person-years (py) in 2007 to 11.79/1000 py in 2013, followed by a plateau for the next 3 years and then an increase to $12.50 / 1000$ py in mid-2017. In females aged $\geq 65$ years, fracture rates declined from $27.49 / 1000$ py in 2007 to $22.08 / 1000$ py in 2013 , then increased to $24.92 / 1000$ py in mid-2017. Likewise, fracture rates in males aged $\geq 65$ years declined from 2007 (12.00/1000 py) to $2013(10.72 / 1000$ py), then increased to 12.04/1000 py in mid-2017. The age- and sex-adjusted fracture rates for most fracture sites declined from 2007 to 2013 by $3.7 \%$ per year $(P=0.310)$. Conclusions Following a consistent decline in fracture rate from 2007 to 2013, trends from 2014 to 2017 indicate fracture rates are no longer declining and, for some fracture types, rates are rising.
\end{abstract}

Keywords Commercial insurance $\cdot$ Fracture trends $\cdot$ Medicare advantage $\cdot$ Osteoporosis

\section{Introduction}

Osteoporosis is an asymptomatic disease characterized by low bone density, poor bone quality, and reduced bone strength and is associated with high risk of fracture [1]. The burden of osteoporotic fractures is significant, including functional

Electronic supplementary material The online version of this article (https://doi.org/10.1007/s00198-020-05334-y) contains supplementary material, which is available to authorized users.

\section{S.A. Williams}

swilliams@ radiuspharm.com

1 New Mexico Clinical Research \& Osteoporosis Center, 300 Oak Street NE, Albuquerque, NM 87106, USA

2 Optum, 11000 Optum Circle, Eden Prairie, MN 55344, USA

3 Radius Health, Inc., 950 Winter Street, Waltham, MA 02451, USA

4 University of Alabama at Birmingham, 510 20th Street South, Faculty Office Towers 802D, Birmingham, AL 35294, USA decline, loss of independence, substantial economic impact, and increased morbidity and mortality [2, 3]. Hip fractures tend to be the most costly and debilitating of fractures, with approximately 10 to $20 \%$ mortality in the year following a hip fracture and more than half of patients unable to return to independent living post-fracture. Fractures at other skeletal sites, including the spine and wrist, are also associated with substantial disability and functional decline [3, 4]. Specifically, Medicare patients over 65 years of age with a spine fracture have been shown to have approximately two times the overall mortality of matched controls, with survival rates consistently significantly lower at 3,5, and 7 years postfracture [5]. Mortality rates were significantly greater for men than women and relative differences in mortality in patients with a spine fracture versus controls was greatest in younger patients (aged 65-69 years) and declined as age at time of fracture increased [5].

Despite the burden of osteoporosis and associated fractures, guidelines for osteoporosis screening and treatment, and the availability of therapeutic options to reduce fracture 
risk $[6,7]$, in recent years, there have been declines in screening and treatment [8-10]. A potential reason for these declines may be decreased availability of office-based dual-energy Xray absorptiometry (DXA), which is possibly due in part to reimbursement reductions by Medicare. In addition, patient safety concerns regarding the use of bisphosphonates (osteonecrosis of the jaw, atypical femur fractures) $[9,11]$ has limited treatment.

Several studies have examined hip fracture trends in the USA. A study by Brauer et al. showed a steady decline in hip fracture incidence from 1995 to 2005 in men and women after a previous rise in rates from 1986 to 1995 in a Medicare population [12]. A recent study by Lewiecki et al. found that US hip fracture rates in the Medicare population declined between 1995 and 2012 but then plateaued in 2013, with a higher than projected incidence in 2013-2015 [13].

The current analysis expands on the fracture incidence trends reported by Lewiecki et al. by evaluating fracture incidence in a younger, commercially insured and Medicare Advantage US population using more recent data from 2007 to 2017. The current study also includes the evaluation of fracture incidence for other skeletal sites, in addition to hip.

\section{Methods}

\section{Data source and study population}

Administrative medical and pharmacy claims data from the Optum $^{\mathrm{TM}}$ Research Database (ORD) were used to identify fracture rates during the period January 1, 2007, to May 31, 2017, in US commercial (private, for-profit) and Medicare Advantage (privately maintained, government-approved) health plan members. Data for approximately 66.3 million commercial and an additional 7.4 million Medicare Advantage enrollees (both men and women) were included in the ORD as of 2017. All data were maintained in a deidentified manner and were accessed following protocols compliant with the Health Insurance Portability and Accountability Act (HIPAA).

Patients aged $\geq 50$ years with evidence of a "case-qualifying" fracture in the identification period (January 1, 2007, to May 31, 2017) were included in the analysis, based on a validated claims-based algorithm $[14,15]$ with a positive predictive value that exceeds 90\% [9]. Case-qualifying fractures were those identified during an inpatient stay (in any position on the medical claim) or in an outpatient setting accompanied by a fracture repair procedure code (e.g., surgery, kyphoplasty). Fracture diagnoses were based on primary or secondary International Statistical Classifications of Diseases and Related Health Problems, 9th Revision (ICD-9) or 10th Revision (ICD-10), listed on the same claim. Exclusion criteria included Paget's disease of bone or malignancy (except non-melanoma skin cancer) at baseline (during the 12 months prior to index fracture) or during the first month of follow-up.

The first fracture during the identification period (January 1,2007 , to May 31,2017 ) was considered the index fracture. Fracture of the spine, pelvis, shoulder, radius/ulna, carpal/ wrist, hip, femur, tibia/fibula, ankle, and multiple sites in the above grouping were included in the analysis. Fractures that occurred within 30 days of the index date were considered index fractures (i.e., part of the initial episode) and defined as fractures at multiple sites; fractures at different sites occurring more than 30 days after the index date were considered subsequent fractures. Episodes continued until a gap of 90 days was observed between consecutive claims.

\section{Study analyses}

An analysis was conducted to examine the trend in fracture rate over time. Age- and sex-adjusted fracture rates were calculated using the age and sex distribution of patients with an index date in 2016. The year 2016 was used because the observation period for 2017 was not a complete year. The fracture rate (number of fractures/1000 years of continuous enrollment) within each year was stratified by age and sex, then weighted to match the age and sex distribution observed in 2016. These stratified rates were condensed by year to generate a yearly age- and sex-adjusted fracture rate. The raw fracture rate for 2016 was equal to the age- and sex-adjusted fracture rate.

Incidence rate was reported as the number of events per 1000 person-years (py) of enrollment during each year of the identification period. The denominator population comprised of members who were $\geq 50$ years of age during the year of interest. The number of days the member was enrolled in the health plan during the year was calculated and was used to determine the total person-years (py) of enrollment for the denominator. Incidence rate is reported as number of events per 1000 py of enrollment during each year from 2007 to May 2017. Fracture rates were stratified by age category (50 to 64 vs. $\geq 65$ years), sex (male vs. female), and fracture site (ankle, pelvis, radius/ulna, hip, shoulder, carpal/ wrist, tibia/fibula, spine, femur, and multiple sites).

In order to test if there was a different trend over time in the rate of fractures between 2007 and 2013 vs. 2014 and 2017, we constructed generalized linear Poisson models with log link. We used three models: (1) females aged 65+ years, (2) males aged $65+$ years, (3) all patients adjusted for age category $(50-54,55-59,60-64,65-69,70-74,75-79,80-84$, and $85+$ years) and sex. For each model, data were aggregated for the unique combinations of calendar year, sex, and age category. The dependent variable was the sum of fractures within each of the combinations. The independent variables were calendar year for years 2007 to 2013 and a separate 
Fig. 1 Fracture rate per 1000 person-years by age and sex which includes fractures of the ankle, carpal/wrist, hip, femur, pelvis, radius/ulna, shoulder, spine, tibia/fibia, or multiple sites

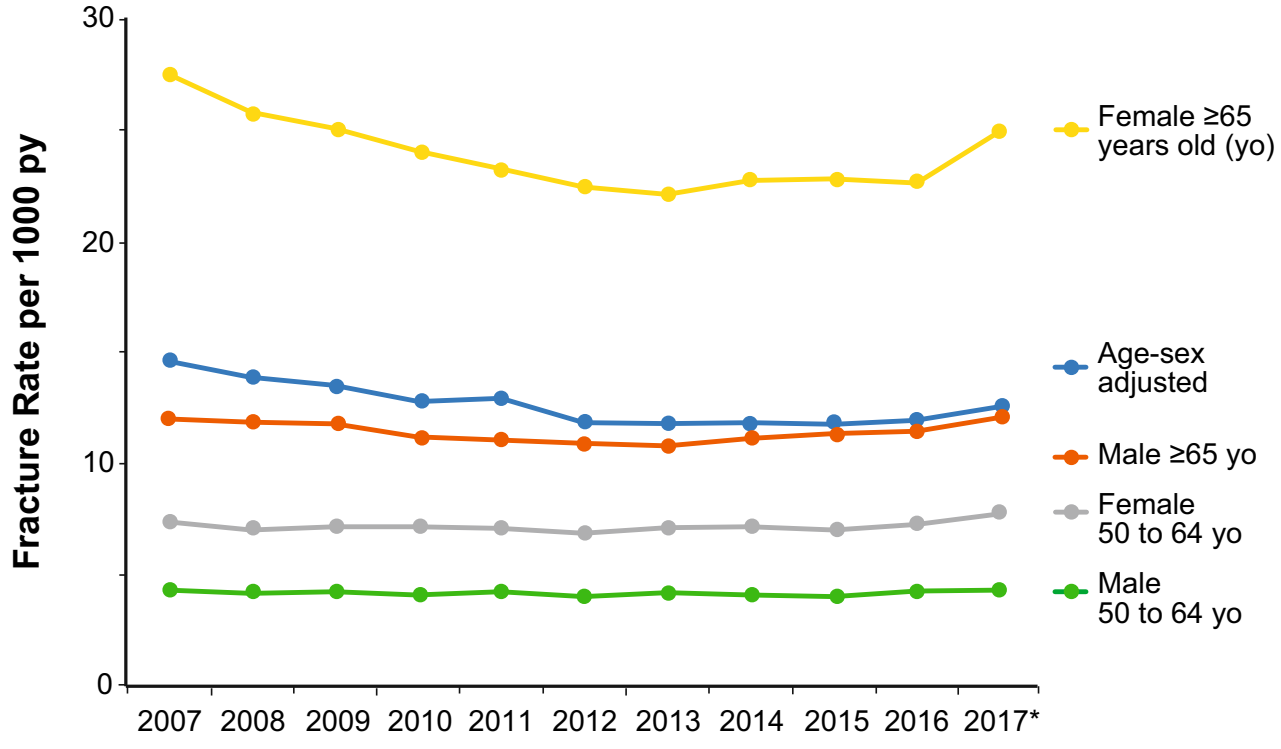

*Up to May 31, 2017; py, person-years. continuous variable for years 2014 to 2017, which allowed for an estimation of the rate ratio for each of the two time periods. The exposure (denominator) was the number of person-years of continuous enrollment in a qualifying medical plan for each of the combinations. Robust standard errors were used to calculate the statistical significance of the rate ratios. A rate ratio less than 1 indicates a reduction in fracture rates over time, while a rate ratio greater than 1 indicates an increase in fracture rates over time. Interpretation for each row is provided with the row.

\section{Results}

\section{Patient identification}

There were 1,841,263 patients identified with an index fracture between January 1, 2007 and May 31, 2017. Of these, 930,690 had case-qualifying fractures and 513,176 had casequalifying fractures in patients aged $\geq 50$ years.

\section{Fracture rates: 2007 to 2017}

The overall age- and sex-adjusted fracture rate fell from 14.67/ 1000 py in 2007 to $11.79 / 1000$ py in 2013 (Fig. 1). Subsequently, the age- and sex-adjusted fracture rate plateaued $(11.80 / 1000$ py in $2014,11.75 / 1000$ py in 2015 , and $11.89 /$ 1000 py in 2016). In 2017 (data as of May 31, 2017), ageand sex-adjusted fracture rate increased to $12.50 / 1000 \mathrm{py}$. Overall, the trend for the age- and sex-adjusted rate from 2007 to 2013 decreased by $3.7 \%$ per year $(P=0.310)$. The fracture rate trend from 2014 to 2017 was $5.3 \%$ higher per year relative to the trend observed from 2007 to 2013, though the difference was not statistically significant (95\% confidence interval [CI] 0.897-1.237; $P=0.527$ ). The rate of fractures from 2014 to 2017 was significantly greater than the rate observed between 2007 and 2013 (rate ratio 1.06). The actual fracture rate between 2014 and 2017 was $2.4 \%(0.966 \times 1.060=1.024)$.

Females $\geq 65$ years of age had the highest rate of fracture among the subgroups analyzed (Fig. 1). Fracture rate declined in this population from $27.49 / 1000$ py in 2007 to 22.08 / 1000 py in 2013. After 2013, fracture rates plateaued (22.74/ 1000 py in $2014,22.81 / 1000$ py in 2015 , and $22.66 / 1000$ py in 2016). As of May 31, 2017, fracture rate increased to 24.92/ 1000 py. The trend in the fracture rate from 2007 to 2013 showed a statistically significant decrease of $3.4 \%$ per year (95\% CI $0.960-0.971, P<0.001$ ). The trend in the fracture rate for the 2014-2017 time period was $6.0 \%$ higher than the annual trend from 2007 to 2013 (95\% CI: 1.04-1.08; $P<0.001)$ indicating a significant change in the fracture rate trend from 2007 to 2013 vs. 2014 to 2017. Among males aged $\geq 65$ years, fracture rate also declined steadily from 2007 (12.00/1000 py) to 2013 (10.72/1000 py). ${ }^{1}$ Subsequently, fracture rate increased each year in males aged $\geq 65$ years $(11.08 / 1000$ py in $2014,11.29 / 1000$ py in 2015 , and $11.39 /$ 1000 py in 2016) to $12.04 / 1000$ py as of May 31,2017 . The trend in the fracture rate from 2007 to 2013 showed a statistically significant decrease of $2.0 \%$ per year $(95 \%$ CI $0.977-$ $0.983, P<0.001)$. The trend in the fracture rate for the 2014 2017 time period was $4.8 \%$ higher than the annual trend from 2007 to 2013 (95\% CI 1.04-1.06; $P<0.001)$. Fracture rate was lowest in patients aged 50 to 64 years and was mostly linear in males and females in this age category.

\footnotetext{
${ }^{1}$ Optum Radius Osteo Report 16 Aug 2018 Appendix B, imbedded worksheet (fracture rate trend) Table 4_checked AL
} 
The spine was the most common site of fracture, followed by the radius/ulna, the hip, and multiple skeletal sites. The age- and sex-adjusted fracture rates for most fracture sites declined from 2007 to 2013 (Fig. 2). Between 2014 and 2017 , the trend in fracture rate varied by site. Rates of ankle fracture (1.30/1000 py in 2014 and $0.88 / 1000$ py in 2017) and pelvis fracture $(0.38 / 1000$ py in 2014 and $0.26 / 1000$ py in $2017)$ continued to decline. Rates of radius/ulna $(2.02 /$ 1000 py in 2014 and $2.01 / 1000$ py in 2017), hip (1.77/ 1000 py in 2014 and $1.75 / 1000$ py in 2017$)$, and carpal/ wrist fracture $(0.10 / 1000$ py in 2014 and $0.11 / 1000$ py in 2017 ) in 2017 were each close to their 2014 values. Conversely, there was a numerical increase in the rates of spine $(2.77 / 1000$ py in 2014 and $3.44 / 1000$ py in 2017), shoulder (1.38/1000 py in 2014 and $1.47 / 1000$ py in 2017$)$, femur $(0.21 / 1000$ py in 2014 and $0.29 / 1000$ py in 2017$)$, and tibia/fibula $(0.35 / 1000$ py in 2014 and $0.53 / 1000$ py in 2017$)$ fracture from 2014 to 2017 . The rate of patients having fractures at multiple sites also numerically increased during this time period (1.52/1000 py in 2014 and $1.78 / 1000$ py in 2017$)$. Additional data are given in the Online Resource Fracture Rate Tables.

\section{Discussion}

This retrospective database study found that age- and sexadjusted fracture rates in the USA have either plateaued or increased from 2014 through mid-2017, in a population of commercially insured and Medicare Advantage health plan enrollees, following a decline from 2007 to 2013. Similar trends were seen in both men and women aged $\geq 65$ years, although overall fracture rates were higher in women, and the increase in fracture rate between 2016 and mid-2017 appeared greater in women aged $\geq 65$ years. Conversely, fracture rates were largely linear during the time period studied in younger women and men (aged 50 to 64 years). Finally, trends in fracture incidence were different depending on the site of fracture.

These findings are consistent with previous studies that identified a decline in hip fracture rates from 1995 to 2012 $[12,13]$. Likewise, our findings support the observation by Lewiecki et al. that the declining trends in hip fracture rates in Medicare enrollees, observed over calendar years 2002 to 2015, have plateaued and started to increase beginning in 2013 [13]. Our study included both commercial and Medicare Advantage enrollees, expanding the finding of Lewiecki et al. to a broader population. We also included fracture sites other than hip in our analysis. Fracture rates at the radius/ulna and carpal/wrist also plateaued from 2014 through mid-2017 after a previous decline, suggesting this increasing trend is not limited to hip fracture rate. Of note, there was an observed increase in the rates of spine, shoulder, femur, tibia/fibula, and multiple-site fractures from 2014 through mid-2017, which has not been previously reported. The spine was the most common site of fracture in this study and also the site with the most pronounced increase from 2014 through mid-2017. Studies suggest that spine fractures are the biggest predictors of future fracture risk, so an increase in spine fractures may lead up to an increase in fractures at other sites in the future [16].

The observed plateau or increase in fracture rates has occurred despite the availability of treatments approved for fracture risk reduction [17]. Although causality for the secular trends cannot be established from observational data, the observed fracture trend may be related to the declining trend in
Fig. 2 Age- and sex-adjusted fracture rates per 1000 personyears by fracture site

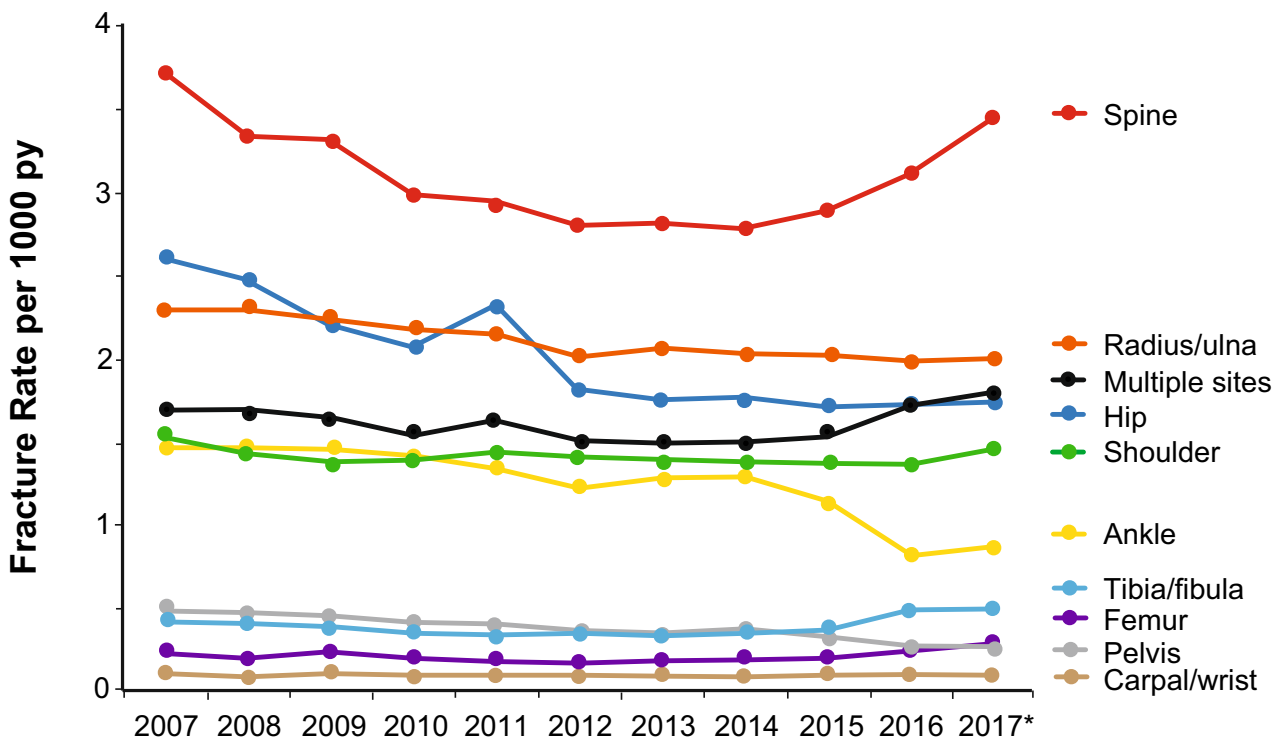

*Up to May 31, 2017; py, person-years. 
osteoporosis-related prescriptions and DXA screening [8-11]. The alarming decline in the diagnosis and treatment of osteoporosis has been recognized as a crisis in the care of osteoporosis [18], and an urgent global call to action was initiated to define strategies to narrow the treatment gap and reduce the burden of osteoporotic fractures [19].

Limitations of this study include those inherent to analyses of claims-based data. Diagnosis and procedure codes were used to identify case-qualifying fractures, but these were not confirmed with imaging studies, although this method has been shown in validation studies to have good accuracy compared with medical record review [20]. Further limitations include the population studied, as only Medicare Advantage enrollees were assessed. These patients are usually less ill than patients on other supplemental Medicare insurance plans, and therefore, the rates of fracture could be underestimated in a more heterogeneous population of patients older than 65 years. Additionally, only clinical spine fractures (those resulting in a visit and generation of a medical claim) were included, potentially further underestimating the incidence of spine fracture events.

\section{Conclusions}

Age- and sex-adjusted US fracture rates have plateaued or increased beginning in 2014 in parallel with the observed population-based declines in osteoporosis screening and treatment. This supports the global call to action to increase screening and treatment of osteoporosis in older adults.

Acknowledgments Funding for this study was provided by Radius Health, Inc., Waltham, MA. All listed authors meet the criteria for authorship set forth by the International Committee for Medical Journal Editors. Medical writing and editorial support (Sarah Hummasti, $\mathrm{PhD}$ ) and graphic services were provided by AOIC, LLC, and were funded by Radius Health, Inc.

\section{Compliance with ethical standards}

Conflicts of interest EML has received no direct income from potentially conflicting entities. His employer, New Mexico Clinical Research \& Osteoporosis Center, has received research grants from Radius, Amgen, Mereo BioPharma, and Bindex; income for service on scientific advisory boards or consulting for Amgen, Radius Health Inc., Alexion Pharmaceuticals, Sandoz, and Samsung Bioepis; service on speakers' bureaus for Radius Health Inc. and Alexion; project development for University of New Mexico; and royalties from UpToDate for sections on DXA, fracture risk assessment, and prevention of osteoporosis. He is a board member of the National Osteoporosis Foundation, International Society for Clinical Densitometry, and Osteoporosis Foundation of New Mexico. BC and KS are employees of Optum, which received funding from Radius Health, Inc., to conduct the study. SAW, RJW, and YW, are employees of and own equity stock in Radius Health, Inc. LAF is a former employee of, is a current consultant, and owns equity stock in Radius Health Inc. JRC is a consultant for Amgen and Radius Health, Inc., and is an employee of the University of Alabama at Birmingham, which has received research grants from Amgen and Radius Health, Inc.
Patient confidentiality All patient data obtained for this analysis were maintained in a de-identified manner and were accessed following protocols compliant with the Health Insurance Portability and Accountability Act (HIPAA).

For this type of study, formal consent is not required.

Open Access This article is licensed under a Creative Commons Attribution-NonCommercial 4.0 International License, which permits any non-commercial use, sharing, adaptation, distribution and reproduction in any medium or format, as long as you give appropriate credit to the original author(s) and the source, provide a link to the Creative Commons licence, and indicate if changes were made. The images or other third party material in this article are included in the article's Creative Commons licence, unless indicated otherwise in a credit line to the material. If material is not included in the article's Creative Commons licence and your intended use is not permitted by statutory regulation or exceeds the permitted use, you will need to obtain permission directly from the copyright holder. To view a copy of this licence, visit http:// creativecommons.org/licenses/by-nc/4.0/.

\section{References}

1. Camacho PM, Petak SM, Binkley N, Clarke BL, Harris ST, Hurley DL, Kleerekoper M, Lewiecki EM, Miller PD, Narula HS, PessahPollack R, Tangpricha V, Wimalawansa SJ, Watts NB (2016) American Association of Clinical Endocrinologists and American College of Endocrinology clinical practice guidelines for the diagnosis and treatment of postmenopausal osteoporosis-2016. Endocr Pract 22(Suppl 4):1-42

2. Cauley JA (2013) Public health impact of osteoporosis. J Gerontol A Biol Sci Med Sci 68(10):1243-1251

3. Dempster DW (2011) Osteoporosis and the burden of osteoporosisrelated fractures. Am J Manag Care 17(Suppl 6):S164-S169

4. Edwards BJ, Song J, Dunlop DD, Fink HA, Cauley JA (2010) Functional decline after incident wrist fractures - study of osteoporotic fractures: prospective cohort study. BMJ 341:c3324

5. Lau E, Ong K, Kurtz S, Schmier J, Edidin A (2008) Mortality following the diagnosis of a vertebral compression fracture in the Medicare population. J Bone Joint Surg Am 90(7):1479-1486

6. ISCD (2015) 2015 official positions - adult. International Society for Clinical Densitometry website. http://www.iscd.org/officialpositions/2015-iscd-official-positions-adult/ Accessed 29 May 2019

7. Preventive Services Task Force US, Curry SJ, Krist AH, Owens DK, Barry MJ, Caughey AB, Davidson KW, Doubeni CA, Epling JW Jr, Kemper AR, Kubik M, Landefeld CS, Mangione CM, Phipps MG, Pignone M, Silverstein M, Simon MA, Tseng CW, Wong JB (2018) Screening for osteoporosis to prevent fractures: US Preventive Services Task Force recommendation statement. JAMA 319(24):2521-2531

8. Hayes BL, Curtis JR, Laster A, Saag K, Tanner SB, Liu C, Womack C, Johnson KC, Khaliq F, Carbone LD (2010) Osteoporosis care in the United States after declines in reimbursements for DXA. J Clin Densitom 13(4):352-360

9. Kim SC, Kim DH, Mogun H, Eddings W, Polinski JM, Franklin JM, Solomon DH (2016) Impact of the U.S. Food and Drug Administration's safety-related announcements on the use of bisphosphonates after hip fracture. J Bone Miner Res 31(8):15361540

10. Solomon DH, Johnston SS, Boytsov NN, McMorrow D, Lane JM, Krohn KD (2014) Osteoporosis medication use after hip fracture in U.S. patients between 2002 and 2011. J Bone Miner Res 29(9): 1929-1937 
11. King AB, Fiorentino DM (2011) Medicare payment cuts for osteoporosis testing reduced use despite tests' benefit in reducing fractures. Health Aff (Millwood) 30(12):2362-2370

12. Brauer CA, Coca-Perraillon M, Cutler DM, Rosen AB (2009) Incidence and mortality of hip fractures in the United States. JAMA 302(14):1573-1579

13. Lewiecki ME, Wright NC, Curtis JR, Siris E, Gagel RF, Saag KG, Singer AJ, Steven PM, Adler RA (2018) Hip fracture trends in the United States, 2002 to 2015. Osteoporos Int 29(3):717-722

14. Bynum JPW, Bell JE, Cantu RV, Wang Q, McDonough CM, Carmichael D, Tosteson TD, Tosteson ANA (2016) Second fractures among older adults in the year following hip, shoulder, or wrist fracture. Osteoporos Int 27(7):2207-2215

15. Curtis J, Chastek B, Sudquist K, Barrera-Sierra S, Leader D, Weiss R, Wang Y, Williams S (2018) Economic burden of osteoporosisrelated fractures in a population of U.S. managed care enrollees. J Manag Care Spec Pharm 24(Suppl 10a):S84

16. Kendler DL, Bauer DC, Davison KS, Dian L, Hanley DA, Harris ST, McClung MR, Miller PD, Schousboe JT, Yuen CK, Lewiecki
EM (2016) Vertebral fractures: clinical importance and management. Am J Med 129(2):221.e1-221e10

17. Khosla S, Hofbauer LC (2017) Osteoporosis treatment: recent developments and ongoing challenges. Lancet Diabetes Endocrinol 5(11):898-907

18. Khosla S, Shane E (2016) A crisis in the treatment of osteoporosis. J Bone Miner Res 31(8):1485-1487

19. Dreinhofer KE, Mitchell PJ, Begue T, Cooper CML, Falaschi P, Hertz K, Marsh D, Maggi S, Nana A, Palm H, Speerin R, Magaziner J (2018) A global call to action to improve the care of people with fragility fractures. Injury 49(8):1393-1397

20. Wright NC, Daigle SG, Melton ME, Delzell ES, Balasubramanian A, Curtis JR (2019) The design and validation of a new algorithm to identify incident fractures in administrative claims data (2019). J Bone Miner Res. 2019 Jun 6. https://doi.org/10.1002/jbmr.3807. [Epub ahead of print]

Publisher's note Springer Nature remains neutral with regard to jurisdictional claims in published maps and institutional affiliations. 